\title{
¿Determinísticamente probable o probabilísticamente determinista?
}

\author{
G. M. Ortigoza ${ }^{1}$ - A. Lorandi ${ }^{1}$
}

\begin{abstract}
RESUMEN
Este trabajo presenta la revisión de algunos modelos que conocemos como determinísticos o como estocásticos, así como algunas relaciones entre ellos, las cuales resultan interesantes. Vemos cómo las caminatas aleatorias generan algunas ecuaciones diferenciales parciales tales como la ecuación de calor; se presenta la ecuación de Laplace resuelta usando el juego Tour du wino; es decir, simulación Montecarlo para obtener los valores de una función armónica, como los promedios de su valores en la frontera obtenidos por diferentes trayectorias. Se revisan los modelos de Black and Scholes, así como el método de funciones generadoras de probabilidad para mostrar como determinados problemas probabilísticos pueden resolverse usando métodos determinísticos basados en ecuaciones diferenciales ordinarias y parciales.
\end{abstract}

\begin{abstract}
This work presents a review of some deterministic and stochastic models, interesting relationships between them, are also discussed. Random walks give rise to partial differential models such as the heat equation. A Tour du wino game is introduced to approximate a solution to Laplace equation, here a Monte Carlo simulation is used to obtain the values of an harmonic function as the average of its boundary values using random trajectories. We review the Black $\&$ Scholes and the probability generating functions models to show how some probabilistic problems can be solved using deterministics methods (based on ordinary and partial differential equations).
\end{abstract}

Palabras Clave: Modelos determinísticos, modelos probabilísticos, Montecarlo, caminatas aleatorias, Black and Scholes

Keywords: BDeterministic models, Stochastic models, Monte Carlo, random walk, Black and Scholes

1 Facultad de Ingeniería de la Universidad Veracruzana, Boca del Río, Veracruz. Contacto: gerardo_ortigoza@yahoo. com 
Introducción

巨 n la dinámica del punto material, la ecuación de movimiento (ecuación diferencial) determina la posición futura de un objeto o partícula móvil en función de otras variables como, su velocidad, su aceleración, su masa y cuantas variables le pueden afectar en su movimiento junto con las condiciones iniciales. Así el futuro a partícula está completamente determinado por su pasado y decimos que nuestro modelo es determinístico.

Existe otro tipo de modelos en los cuales hay cierta incertidumbre en la predicción del resultado de un proceso. En este caso nos referimos a un proceso aleatorio y llamamos probabilísticos a los modelos correspondientes.

Cabe mencionar en este punto el caso de la teoría del caos, que estudia determinados tipos de sistemas complejos y sistemas dinámicos muy sensibles a las variaciones en las condiciones iniciales. Pequeñas variaciones en dichas condiciones iniciales pueden implicar grandes diferencias en el comportamiento futuro, imposibilitando la predicción a largo plazo. Esto sucede aunque estos sistemas son en rigor deterministas. Este hecho ha sido usado para generar números pseudoaleatorios usando sistemas dinámicos [12].

En el caso de interacción de muchas partículas es común adoptar un modelo en el que el movimiento de cada partícula se describe mediante ciertas probabilidades. Tanto procesos determinísticos como los procesos aleatorios por sí mismos son de considerable importancia; sin embargo, en este trabajo se presentan algunas de las conexiones entre modelos probabilísticos y determinísticos. Aun cuando un proceso es considerado como aleatorio, en algunos casos podemos escribir problemas determinísticos en ecuaciones diferenciales parciales que dan origen a una función de distribución definida para las probabilidades asociadas con el proceso. La ecuación diferencial parcial para la función de distribución es la misma que obtendríamos en una caracterización determinística del fenómeno. Parece un tanto paradójico que un proceso aleatorio pueda ser caracterizado por una ecuación determinística. Tal es el caso del modelo de Black Scholes, donde mediante el lema de Ito, una ecuación diferencial estocástica se reduce a una ecuación diferencial parcial determinística, que una vez resuelta nos ayuda a valuar el precio de una opción sobre un activo.

Más sorprendente aún es que llegamos a aceptar como aleatorios los números pseudoaleatorios generados por una computadora finita; incluso usamos estos números para resolver problemas determinísticos como el cálculo de una integral, o la ecuación de Laplace con muestreos estocásticos mediante el método de Montecarlo. En este trabajo se revisa algunos modelos que conocemos como determinísticos o como estocásticos así como algunas relaciones entre ellos, las cuales resultan interesantes; el trabajo está organizado de la siguiente manera: en la sección 2 se presentan eventos probabilísticos que generan modelos determinísticos, se introduce la caminata aleatoria y se muestra cómo este proceso aleatorio genera a la ecuación del calor determinística, así también se muestra el método de Montecarlo para resolver (generando trayectorias aleatorias y los promedios de valores en la frontera) el problema de Dirichlet para la ecuación de Laplace; la sección 3 nos presenta ejemplos de problemas probabilísticos que pueden resolverse con modelos determinísticos basados en ecuaciones diferenciales tanto ordinarias como parciales: una ecuación estocástica para el precio de una acción se reduce a una ecuación diferencial determinística, así como también se muestra la solución de un problema idealizado de líneas telefónicas resuelto por el método de funciones generadoras de probabilidad; finalmente en la sección 4 se incluyen algunas conclusiones.

2. Eventos probabilísticos que generan modelos determinísticos

\subsection{Caminata aleatoria y la Ecuación del calor}

Consideremos la caminata aleatoria de una partícula a lo largo de la recta real [4]. Supongamos que la regla del movimiento es tal que a 
cada paso de tiempo de tamaño $\tau$, la partícula brinca a la derecha o a la izquierda una distancia con la misma probabilidad (de 1/2).

Supongamos que al tiempo $t=0$ la partícula está en $\mathrm{x} x=x_{0}$; estamos interesados en obtener la densidad de probabilidad de la posición de la partícula $u(x, t)$.

Consideremos la probabilidad de que la partícula alcance la posición $x$ al tiempo $t+\tau$; de acuerdo a las reglas del movimiento hay dos posibilidades: la partícula estaba en $x-h$ al tiempo $t$ y brincó a la derecha o la partícula estaba en $x+h$ al tiempo $t$ y brincó a la izquierda. Como la posición de la partícula al tiempo $t$ y la dirección de su siguiente movimiento son independientes, la primera situación sucede con probabilidad $\frac{1}{2} u(x+h, t)$ mientras que la segunda con $\frac{1}{2} u(x-h, t)$, con lo que tenemos:

$$
(x, t+\tau)=\frac{1}{2} u(x+h, t)+\frac{1}{2} u(x-h, t)
$$

Podemos usar el teorema de Taylor para expandir ambos lados de la ecuación

$$
u(x, t+\tau)=u(x, t)+u_{t}(x, t)+o(\tau)
$$

$u(x \pm h, \tau)=u(x, t) \pm u_{x}(x, t) h+\frac{1}{2} u_{x x}(x, t) h^{2}+o\left(h^{2}\right)$

Sustituyendo (2) y (3) en (1) obtenemos:

$$
u_{t}+o(1)=\frac{1}{2} \frac{h^{2}}{\tau} u_{x x}+o\left(\frac{h^{2}}{\tau}\right)
$$

Observamos que una dinámica no trivial sólo sucede cuando $\frac{h^{2}}{\tau} \rightarrow 2 D$ para un número positivo finito $D$; así obtenemos la Ecuación del calor o de difusión.

$$
u_{t}(x, t)=D u_{x x}(x, t) .
$$

Otras ecuaciones diferenciales parciales ta- les como la ecuación de adveción-difusión $u_{t}+v u_{x}=D u_{x x} \quad$ o la ecuación del telegrafista $u_{t t}+\alpha u_{t}+\beta u=c^{2} u_{x x} \quad$ pueden obtenerse usando caminatas aleatorias ([9], [1]). En el proceso límite de caminatas aleatorias se pueden obtener conjuntos de ecuaciones parciales de tipo parabólico o hiperbólico con soluciones de tipo onda viajera [11].

En resumen hemos observado que un proceso probabilístico como la caminata aleatoria da lugar a una ecuación diferencial parcial determinística.

\subsection{Método de Montecarlo}

El método de Montecarlo permite obtener soluciones aproximadas a una gran variedad de problemas matemáticos mediante la realización de experimentos con muestreos de números pseudoaleatorios generados en una computadora. El método es aplicable a una amplia gama de problemas tanto estocásticos como deterministas. A diferencia de los métodos numéricos que se basan en evaluaciones en $N$ puntos en un espacio $M$-dimensional para producir una solución aproximada, el método de Montecarlo tiene un error absoluto de la estimación que decrece como $1 / \sqrt{n}$ obtenido mediante el Teorema del límite central. El método de Montecarlo se usa en finanzas y matemáticas financieras para evaluar y analizar instrumentos complejos como portafolios e inversiones, al simular las diferentes fuentes de incertidumbre que afectan su valor y entonces determinar su valor promedio sobre el rango de resultados posibles. Las ventajas de Montecarlo sobre otros métodos se incrementan cuando el número de las dimensiones (fuentes de incertidumbre) del problema crece ([5], [7]).

Consideremos el problema de Dirichlet para la ecuación de Laplace:

$$
\begin{gathered}
\Delta u=0 \quad 0<x, y<1 \\
u(x, y)=g(x, y)= \begin{cases}1 & y=1,0<x<1 \\
0 & \text { deotra forma }\end{cases}
\end{gathered}
$$


Definamos las reglas de un juego aleatorio (Tour du wino)

Paso 1 Iniciamos de cualquier punto interior A .

Paso 2 En cada etapa del juego este punto se mueve de manera aleatoria hacia cualquiera de sus puntos vecinos con probabilidad 1/4.

Paso 3 Después de llegar a un punto vecino, continua moviéndose hasta llegar a un punto $p_{i}$ de la frontera; paramos y guardamos el punto; esto completa una caminata aleatoria.

Paso 4 Repetimos los pasos 1 al 3 hasta tener suficientes caminatas aleatorias. Ahora calculamos la fracción de veces que incidimos en el punto $p_{i}$ de la frontera.

Paso 5 Supongamos que asignamos a cada punto $p_{i}$ un valor $g_{i}$, asumimos que el juego asigna el valor promedio de las caminatas. El valor asignado a $A$ es

$$
R(A)=g_{1} P_{A}\left(p_{i}\right)+g_{2} P_{A}\left(p_{2}\right)+\ldots+g_{n} P_{A}\left(p_{n}\right)
$$

El valor promedio obtenido resulta ser una solución aproximada del problema de Dirichlet.

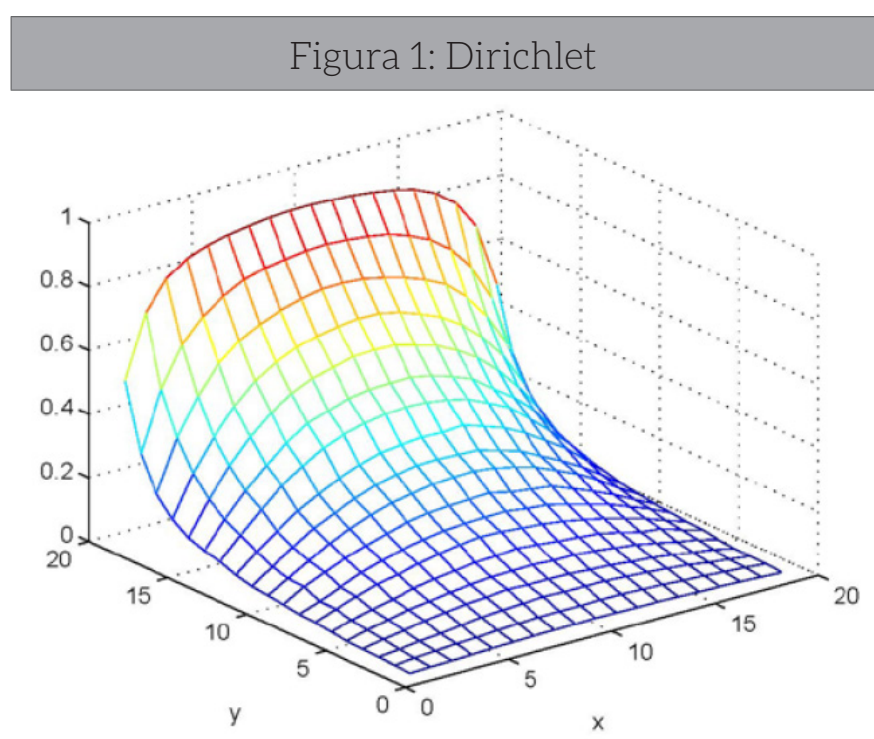

La Figura 1 muestra la solución aproximada del problema de Dirichlet obtenida por simulación Montecarlo. En un contexto más general, algunos problemas clásicos de valores en la frontera pueden resolverse usando una representación integral equivalente, donde las trayectorias de caminatas aleatorias son simuladas en la frontera del dominio como cadenas de Markov generadas por el núcleo de la representación integral. Con las ventajas de: 1) poder resolver problemas de muchas dimensiones, 2) es un método libre de malla y da la solución simultánea en cualquier punto, 3) se aplica a problemas internos o externos, 4) las implementaciones en paralelo son más sencillas [6].

3. Modelos determinísticos que resuelven problemas estocásticos

\subsection{El Modelo de Black and Scholes}

Consideremos una opción, la cual es un contrato que da a su poseedor el derecho (pero no la obligación) de comprar o vender algún bien tal como una cantidad de acciones del mercado bursátil, en una fecha específica (digamos al tiempo $T$ ) cuando el precio de ejercicio $E$ (cantidad previamente pactada) es pagado por el bien [2].

Supongamos que el bien al que nos referimos es una acción la cual se espera que aumente en valor en el intervalo de tiempo $0<t<T$, pero es tal que su precio está sujeto a fluctuaciones impredecibles. Supongamos que compramos una opción en lugar de la acción; obtendremos una ganancia si la acción aumenta su precio y una pérdida si lo disminuye (ya que no tendría sentido pagar $E$ por algo que cuesta menos que eso en el mercado). Sin embargo, podemos proteger la posición de opción definiendo un portafolio de la opción y un cierto número de acciones. El contar con las acciones nos permitirá protegernos en contra de lo impredecible y este proceso nos permitirá calcular el valor $V(S, t)$, de la opción de comprar una acción al tiempo $T$ como una función del tiempo $t$ y del valor de la acción $S$. Supongamos que la tasa de ganancia instantánea en la acción varía aleatoriamente:

$$
\frac{d S}{S}=\mu d t+\sigma d X
$$

donde ces una tasa de crecimiento determi- 
nística de la acción, $d X$ es una variable normal aleatoria pequeña de media cero y varianza $d t$ que modela la respuesta incierta del precio de la acción a la llegada de nueva información, y $\sigma$ es un parámetro que mide la volatilidad del precio de la acción. Usando el lema de Ito [1] podemos estimar el cambio en $V$ como,

$d V=\left(\sigma S \frac{\partial V}{\partial S} d X\right)+\left(\frac{\partial V}{\partial T}+\mu S \frac{\partial V}{\partial S}+\frac{1}{2} \sigma^{2} S^{2} \frac{\partial^{2} V}{\partial S^{2}}\right) d t$

La tasa de interés libre de riesgo $r$ y la volatilidad $\sigma$ del activo se suponen constantes durante el tiempo que dura la opción. No hay costos de transacción asociados a la cobertura del portafolio. El activo subyacente no paga dividendos durante la vida de la opción. No hay posibilidad de arbitraje. La ausencia de arbitraje significa que todos los portafolios

libres de riesgo deben tener el mismo retorno. La compra y venta del activo puede tomar lugar continuamente. La venta y los activos son divisibles. Asumimos que podemos comprar y vender cualquier número (no necesariamente entero) del activo subyacente y que está permitido vender aunque no tengamos posesión; es decir, se trata de un mercado completo [1].

Vamos a definir un portafolio libre de riesgo.

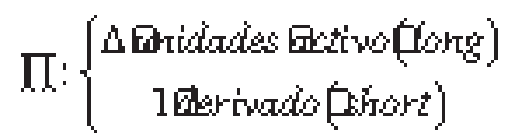

$$
\begin{aligned}
& \Pi=S \Delta-V \\
& d \Pi=d S \Delta-d V=(S \mu d t+S \sigma d X) \Delta-d V \\
& d \Pi=(S \mu d t+S \sigma d X) \Delta-\left(\sigma S \frac{\partial V}{\partial S} d X+\frac{\partial V}{\partial t}+\mu S \frac{\partial V}{\partial S}+\frac{1}{2} \sigma^{2} S^{2} \frac{\partial^{2} V}{\partial S^{2}}\right) d t \\
& d \Pi=\left(\Delta \sigma S-\sigma S \frac{\partial V}{\partial S}\right) d X+\left(\Delta \mu S-\frac{\partial V}{\partial t}-\mu S \frac{\partial V}{\partial S}-\frac{1}{2} \sigma^{2} S^{2} \frac{\partial^{2} V}{\partial S^{2}}\right) d t
\end{aligned}
$$

$$
d \Pi=-\left(\frac{\partial V}{\partial t}+\frac{1}{2} \sigma^{2} S^{2} \frac{\partial^{2} V}{\partial S^{2}}\right) d t
$$

Por la hipótesis de no arbitraje, como el portafolio es libre de riesgo tenemos que su retorno es igual al de un bono de tasa $r, d \Pi=\Pi r d t=(\Delta S-V) r d t=\left(\frac{\partial V}{\partial S} S-V\right) r d t$, con lo que obtenemos:

$$
\begin{gathered}
\left(\frac{\partial V}{\partial S}-V\right) r d t=-\left(\frac{\partial V}{\partial t}+\frac{1}{2} \sigma^{2} S^{2} \frac{\partial^{2} V}{\partial S^{2}}\right) d t \\
\frac{\partial V}{\partial t}+\frac{1}{2} \sigma^{2} S^{2} \frac{\partial^{2} V}{\partial S^{2}}+r S \frac{\partial V}{\partial S}=r V,
\end{gathered}
$$

así tenemos la ecuación de Black-Sholes una ecuación determinística para obtener $V(S, t)$, el valor de la opción, que depende del valor aleatorio de la acción. Dicho sea de paso, el proceso estocástico inicial;, es decir, la variación del precio de la opción en tiempo y costo está definido en intervalos finitos (el precio cambia en fracciones de dólar y el mercado está abierto en días hábiles trading days) la ecuación obtenida asume cambios continuos en el precio y en el tiempo, más aun regularmente la ecuación diferencial parcial se resuelve usando diferencias finitas; esto es, un proceso discreto. En resumen lo anterior ejemplifica como una ecuación probabilística se resuelve con ayuda de una ecuación diferencial parcial determi- 
Figura 2: Simulación de algunas trayectorias de la variación del precio de un activo

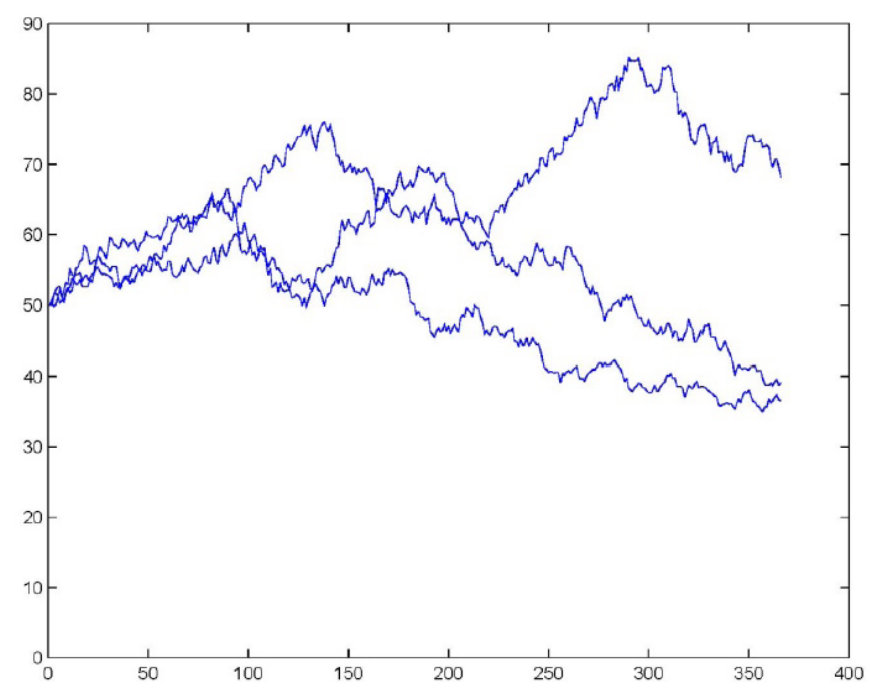

3.2 El método de funciones generadoras de probabilidad

Consideremos una red telefónica idealizada que consiste de un número infinito de líneas [10], y asumamos que las llamadas se originan y se concluyen en la red durante un intervalo de tiempo $[0,1)$ de acuerdo a las siguientes hipótesis:

i) Si una línea está ocupada al tiempo t, la probabilidad de que la conversación termine en el intervalo $(t, t+h)$,es $\mu h+o(h)$ , $(o(h)$ significa que se anula más rápido que $h$ cuando $h \rightarrow 0$ ), donde $\propto$ es una constante.

ii) La probabilidad de que una llamada inicie en el intervalo $(t, t+h)$ es $\lambda h+o(h)$ con $\lambda$ una constante.

iii) La probabilidad de que dos o más cambios ocurrane n el intervalo $(t, t+h)$ es $o(h)$

El problema a resolverse es: Dado un número entero no negativo $n$, hallar la probabilidad $P_{n}(t)$ de que exactamente $n$ líneas estén en uso al tiempo $t, 0<n<\infty$, suponiendo que las probabilidades iniciales $P_{n}(0), 0 \leq n<\infty$ son conocidas.

Supongamos que a un tiempo fijo $t$ se cono- cen $P_{n}(t)$ para todos los $n, 0 \leq n<\infty$ y vamos a determinar $P_{n}(t+h)$, la probabilidad de que $n$ líneas estén en uso al tiempo $t+h$. Para $n \geq 1$ habrá $n$ líneas en uso al tiempo $t+h$ si una de las siguientes condiciones se cumple:

1. Al tiempo $t \quad n$-1 líneas están en uso y una llamada se origina durante el intervalo $(t, t+h)$

2. Al tiempo $t n+1$ líneas están en uso y una llamada se termina durante el intervalo $(t, t+h)$

3. Al tiempo $t n$ líneas están en uso y ningún cambio ocurre en la red durante el intervalo $(t, t+h)$.

4. Dos o más cambios ocurren durante el intervalo $(t, t+h)$.

De acuerdo a estas hipótesis, la probabilidad del evento (4) es $o(h)$; la del evento en (1) es

$$
[\lambda h+o(h)] P_{n-1}(t)
$$

la probabilidad de (2) es:

$$
(n+1)[\mu h+o(h)] P_{n+1}(t)
$$

y la probabilidad de (3) es:

$$
[1-\lambda h-n \mu h+o(h)] P_{n}(t)
$$

y puesto que los eventos son mutualmente exclusivos se tiene que:

$P_{n}(t+h)=\lambda h P_{n-1}(t)+(n+1) \mu P_{n+1}(t)+(1-\lambda h-n \mu h) P_{n}(t)+o(h)$

Usando la expresión anterior escribimos el cociente $\left[P_{n}(t+h)-P_{n}(t)\right] / h$ y calculamos el límite cuando $h \rightarrow 0$ para obtener las ecuaciones diferenciales ordinarias

$P_{n}^{\prime}(t)=-(\lambda+n \mu) P_{n}(t)+\lambda P_{n-1}(t)+(n+1) \mu P_{n+1}(t)$ 
que se cumple para $n \geq 1$. Para $n=0$ un análisis similar nos da:

$$
P_{0}^{\prime}(t)=-\lambda P_{0}(t)+\mu P_{1}(t)
$$

puesto que las probabilidades iniciales $P_{n}(0), 0 \leq n<\infty$ se asumen conocidas; el problema de hallar las probabilidades $P_{n}(t)$ para $t>0$ ha sido reducido al problema de valor inicial para el sistema infinito de ecuaciones diferenciales ordinarias, para resolverlo hallaremos la solución de una ecuación diferencial parcial de primer orden.

Sea $G(t, s)=\sum_{n=0}^{\infty} P_{n}(t) s^{n}$ la función generadora de las probabilidades $P_{n}(t)$. Calculando las derivadas tenemos

$$
G(t, s)=e^{\frac{\lambda}{\mu} S} f\left(e^{-\mu t}(s-1)\right)
$$

la función f está determinada por la condición inicial, haciendo $t=0$ tenemos

$$
\begin{aligned}
& g(s)=e^{\frac{\lambda}{\mu} s} f(s-1) \text { Y así } \\
& \quad f(s)=g(s+1) e^{\frac{-\lambda}{\mu}(s+1)}
\end{aligned}
$$

finalmente substituyendo esta expresiones obtenemos la solución del problema de valor inicial

$$
G(t, s)=g\left(1+e^{-\mu t}(s-1) \exp \left[\frac{\lambda}{\mu}(s-1)\left(1-e^{-\mu t}\right)\right]\right)
$$

Una vez encontrada la función generadora $G(t, s)$ las probabilidades $P_{n}(t)$

Después de substituir la expresión para $P_{n}^{\prime}(t)$ y rearreglando los términos de las series tenemos:

$$
\frac{\partial G}{\partial s}+\mu(s-1) \frac{\partial G}{\partial s}=\lambda(s-1) G
$$

por otra parte el que las condiciones iniciales $P_{n}(0)$ sean conocidas nos da la condición inicial para $G, G(0, s)=g(s)=\sum_{n=0}^{\infty} P_{n}(0) s^{n}$; el sistema de ecuaciones diferenciales ordinarias asociado a la ecuación diferencial parcial es

$$
\frac{d t}{1}=\frac{d s}{\mu(s-1)}=\frac{d G}{\lambda(s-1) G}
$$

y dos primeras integrales funcionalmente independientes son:

$$
u_{1}=e^{-\mu t}(s-1), \quad u_{2}=e^{-\frac{\lambda}{\mu} s} G
$$

puesto que $\boldsymbol{u}_{1}$ no depende de $G$ podemos escribir la integral general como $u_{2} 0 f\left(u_{1}\right)$ para una función $f \in C^{1}$ arbitraria, de donde se obtiene
En [13] la conversión de modelos determinísticos a estocásticos es ilustrada con varios ejemplos representativos que incluye: reacciones reversibles con controles de retroalimentación, cinética enzimática de Michaelis-Menten, etc. Una conexión interesante entre modelos determinísticos y estocásticos para el crecimiento microbiano se encuentra en [3] donde se discuten algunos modelos deterministas de retraso bacteriano y crecimiento subsecuente, así como la relación con modelos que describen la multiplicación bacteriana

debida a procesos estocásticos de nacimiento. Modelos determinísticos basados en ecuacio-

\section{Discusión}


nes diferenciales ordinarias pueden capturar relaciones esenciales entre virus; sin embargo, una infección podría ser iniciada por una sola partícula viral que libera su gnoma, una solo molécula o ADN a la célula que lo alberga. Bajo tales condiciones, un modelo estocástico que permita fluctuaciones inherentes en los niveles de constituyentes virales podría dar un comportamiento cualitativo diferente. Para comparar enfoques de modelación, en [8] se desarrolla un modelo simple de cinética intracelular de un virus gen érico el cual se puede implementar de forma determinística y estocástica.

\section{Conclusiones}

Hemos presentado ejemplos de las relaciones que existen entre modelos determinísticos y probabilísticos. Si bien es cierto que existen conexiones como el hecho de que algunos modelos determinísticos surgen como límites de modelos estocásticos, también hay similitudes pues una colección grande de sistemas tanto determinísticos como estocásticos tiene un comportamiento bastante estable a largo plazo. No debemos ignorar que también existe una colección grande de sistemas determinísticos y estocásticos cuyo comportamiento a largo plazo son bastante diferentes. Al modelar un fenómeno ya sea usando métodos determinísticos o probabilísticos, usualmente uno de los objetivos matemáticos es minimizar el término de error o, y un modelo es a menudo preferido sobre el otro si puede mostrarse que su término de error es menor. Por ejemplo la calibración de un modelo determinista para una cuenca hidrológica, se reduce a minimizar sus elementos estocásticos. Algunas veces, los esfuerzos que se hacen para la validación de modelos deterministas se enfocan en aspectos estocásticos como la preservación del sesgo y la varianza de los caudales. Otras veces los esfuerzos para la validación se enfocan solo en modelos residuales como si el modelo determinístico fuera un modelo de regresión estadística. En términos generales, ingenieros y científicos no han aprendido aún como calibrar o validar modelos determinísticos sin utilizar un análisis de sus componentes estocásticos. Numerosas ventajas surgen de combinar puntos de vista determinísticos y estocásticos, cuando se formulan, prueban o aplican modelos. Una visión del mundo estocástica puede ayudarnos a desarrollar modelos deterministas que puedan representar fielmente las relaciones observadas entre las entradas y los resultados de salida del modelo. Mientras que un enfoque deterministico puede ayudarnos a desarrollar modelos estocásticos que puedan representar fielmente ciertos procesos físicos observados internamente.

Finalmente, si ambas iniciativas son tomadas en cuenta, los modelos no serán ni determinísticos ni estocásticos. En lugar de ello, los modelos balancearan ambas perspectivas, eventualmente conduciendo al surgimiento de modelos más creíbles y 'útiles, con una mejor descripción de la realidad, que es la finalidad de la modelación.

\section{Referencias}

Hull J. C., (2003), Options, Futures \& other Derivatives, Prentice Hall,

Z. Kutalika, Moe Razaza, Jozsef Barany, (2005), Connection between stochastic and deterministic modelling of microbial growth, Journal of Theoretical Biology 232, 285-299.

G. F. Lawler, V. Limic, (2010), Random Walk: A Modern Introduction, Cambridge Studies in Advanced Mathematics.

Lin C. C. \& Segel L.A.,(1995), Mathematics Applied to Deterministic Problems in the Natural Sciences, SIAM.

Sadiku M. N.O., (2009), Monte Carlo Methods for Electromagnetics, CRC Press.

Sabelfeld K. K., \& Simonov, N. A. (2016), Stochastic Methods for Boundary Value Problems: Numerics for High-Dimensional PDEs and applications. Walter de Gruyter $\mathrm{GmbH} \& \mathrm{Co} \mathrm{KG}$.

Alexander Sipin, (2014), Monte Carlo Method for Partial Differential Equations, Chapter book, Topics in Statistical Simulation, Vol 114 of the series Springer Proceedings in Mathematics \& Statistics, pp 465-473. 
Srivastavawz R., Youw L., Summersy J. \& Yin J., (2002), Stochastic vs.Deterministic Modeling of Intracellular Viral Kinetics, J. theor. Biol, vol. 218, 309-321.

Zbigniew J. Grzywna,and Jacek K. Stolarczyk, (2005), Diffusion in Glassy Polymers from Random Walks to Partial Differential Equations, Acta Physica Polonia B, vol 36,No 5.

Mohamed Nageb Elsherbeny, Mahmud Rahal, (2012), Pseudo-Random Number Generator Using Deterministic Chaotic System, International Journal of Scientific and Technol.

Jialiang Wu, Brani Vidakovic and Eberhard Voit, (2005), Constructing stochastic models from deterministic process equations by propensity adjustment, Systems Biology, vol. 5:187.

Zachmanoglou, E. C. \& Thoe, D. W., (1986), Introduction to Partial Differential Equations with Applications. Courier Corporation.

Zauderer, E. (2011), Partial Differential Equations of Applied Mathematics, (Vol 71). John Wiley \& Sons.

Stewart, J. (2011). Calculus. Cengage Learning. 